\title{
Anti-Kasha Conformational Photo-Isomerization of a Heteroleptic Dithiolene Metal Complex Revealed by Ultrafast Spectroscopy
}

Michela Gazzetto, a Flavia Artizzu, ${ }^{b}$ Salahuddin S. Attar, ${ }^{c, \#}$ Luciano Marchiò, a Luca

Pilia, ${ }^{e}$ Egmont J. Rohwer, a Thomas Feurer, a Paola Deplano*, ${ }^{\text {c }}$ Andrea Cannizzo*a

a Institute of Applied Physics, University of Bern, Sidlerstrasse 5, 3012 Bern

(Switzerland)

b L3-Luminescent Lanthanide Lab, Department of Chemistry, Ghent University,

Krijgslaan 281-building S3, B-9000, Gent (Belgium).

c Department of Chemical and Soil Sciences, University of Cagliari, 09042, Monserrato (CA) (Italy).

d Dipartimento di Scienze Chimiche, della Vita e della Sostenibilità Ambientale, Università di Parma, Parco Area delle Scienze 17/a, 43124, Parma (Italy) *corresponding authors: andrea.cannizzo@iap.unibe.ch, deplano@unica.it. 
e Dipartimento di Ingegneria Meccanica, Chimica e dei Materiali, Università di Cagliari,

Via Marengo 2, 09123, Cagliari (Italy).

\# Currently at the Department of Chemistry, Texas A\&M University at Qatar, Texas

A\&M Engineering Building, Education City, Doha, (Qatar)

S1. EMISSION QUANTUM YIELD AND RADIATIVE LIFETIME 
S10. COMPARISON OF THE $S_{2} \rightarrow S_{1}$ INTERNAL CONVERSION AND S24
$S_{2} \rightarrow G S$
LIFETIME WITH LITERATURE 
S1. EMISSION QUANTUM YIELD AND RADIATIVE LIFETIME

Emission Quantum Yield measurements. Emission $\Phi$ was evaluated using the relative method Suzuki ${ }^{1}$ though the following equation:

$$
\Phi=\Phi_{\mathrm{R}} \frac{\mathrm{a}_{\mathrm{R}} \mathrm{In}^{2}}{\mathrm{aI}_{\mathrm{Rn}}{ }_{\mathrm{R}}^{2}}
$$

where the R index refers to the photoluminescence standard, $\Phi_{R}$ is the quantum yield, $a_{R}=10^{-A_{R}}$ is the absorption factor $\left(A_{R}=\right.$ absorbance $)$ at excitation wavelength, $I_{R}$ is the corrected integrated emission and $\mathrm{n}$ is the refractive index of the medium.

For $[\mathrm{Ru}(\mathrm{bpy}) 3]^{2+}$ : Excitation wavelength, $\lambda_{\mathrm{exc}}=450 \mathrm{~nm}$. Emission maximum, $\lambda_{\max }=610$ $\mathrm{nm}$. Concentration was $1.17 \cdot 10^{-6} \mathrm{M}$ in water. For (1a): Excitation wavelength, $\lambda_{\mathrm{exc}}=450$ $\mathrm{nm}$. Emission maximum, $\lambda_{\max }=722 \mathrm{~nm}$. Concentration was $5.00 \cdot 10^{-5} \mathrm{M}$ in acetonitrile solution.

The low emission $\Phi$ of $\sim 10^{-4}$ speaks for a depopulation still dominated by non-radiative deactivation pathways, which strongly quench the radiative de-excitation.

Table S1: Experimental data for the calculation of the $\Phi$.

\begin{tabular}{|l|c|c|c|c|c|c|}
\hline Sample & $\begin{array}{l}\text { Abs } \\
\text { (OD) }\end{array}$ & A $=1-10^{-}$ & $\begin{array}{c}\text { Intensity } \\
\text { (arb.u.) }\end{array}$ & $\mathrm{n}$ & Solvent & $\Phi_{\mathrm{R}}$ \\
\hline $\mathrm{Ru}(\mathrm{bpy}) 3^{2+}$ & 0.016 & 0.036 & $7.8 \times 108$ & 1.333 & $\mathrm{H}_{2} \mathrm{O}$ & 0.063 \\
\hline$(1)$ & 0.12 & 0.24 & $3.1 \times 106$ & 1.344 & $\mathrm{CH}_{3} \mathrm{CN}$ & $5.4 \times 10-$ \\
\hline
\end{tabular}


Radiative lifetime. The radiative lifetime $\left(\mathrm{T}_{\mathrm{rad}}=1 / \mathrm{K}_{\mathrm{rad}}\right.$, where $\mathrm{K}_{\mathrm{rad}}$ is the rate constant for spontaneous emission) was estimated through the Strickler-Berg equation and here reported from reference 2 :

$$
\kappa_{\text {rad }}=\tau_{\text {rad }}^{-1}=8 \pi n^{2} c \frac{1 g}{\left\langle\lambda^{3}\right\rangle g^{\prime}} \int \frac{\sigma(\lambda)}{\lambda} d \lambda
$$

where $\mathrm{n}$ is the refractive index of the medium ( 1.344 for $\mathrm{CH} 3 \mathrm{CN}), \mathrm{c}$ is the speed of light in vacuum and $g, g^{\prime}$ refer to the degeneration of the ground and excited state respectively. $\left\langle\lambda^{3}\right\rangle$ is the mean value of emission wavelength cubed, which takes into account to the $v^{3}$ factor between the spontaneous and stimulated emission Einstein coefficients; $\sigma(\lambda)$ is the absorption cross-section which was estimated through the excitation spectrum normalized to absorption. The retrieved value for the radiative lifetime is $224 \mathrm{~ns}$, yielding an estimated emission decay constant $\mathrm{T}=14 \mathrm{ps}\left(\mathrm{T}=\Phi \mathrm{T}_{\mathrm{rad}}\right)$. These values are compared with the corresponding ones reported in the main text in section S10. 


\section{S2. DATA ANALYSIS}

A detailed explanation of the analysis method can be found in Ref. ${ }^{3}$. Data were corrected for group velocity dispersion (GVD) of the probe pulse. GVD was measured separately in pure solvents and used to correct the TA spectra for time zero shift at different wavelengths. To avoid possible artefacts due to cross-phase modulation, data points from -200 to 200 fs were neglected during the kinetics fitting. Data were analyzed with Singular Value Decomposition (SVD) to reject white noise and decompose the timewavelength signal $(\Delta \operatorname{Abs}(\lambda, A)$ into a minimal number of spectra (so-called Decay Associated Spectra, DAS), each undergoing an exponential decay with its own lifetime?:

$$
\Delta A b s(\lambda, t)=\sum_{i} D A S_{i}(\lambda) \exp \left(-t / \tau_{i}\right) \cdot u(t) \times I R F(t)
$$

where $\times$ represents the convolution operator, $u(t)$ a step function equal to $1(0)$ for $t>0(\leq 0), \quad I R F(t)$ is the time instrumental response function, supposed Gaussian, independent on the probe wavelength, with a width of $130 \mathrm{fs}$ (FWHM) and centered at time zero. In the analysis in the following sections we considered a contribution with $\tau=\infty$, when the corresponding decay was characterized by a time constant much longer than the investigated time interval, namely 250 ps. 
S3. METAL-DITHIOLENE COMPLEXES

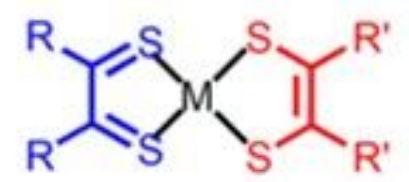

Figure S1: Schematic representations of a donor-acceptor dithiolene metal complex.

A typical feature of these complexes is a low frequency absorption in the visible region, mainly describable as a charge-transfer (CT) transition from the highest occupied molecular orbital (HOMO) -with a predominant contribution of the donor- to the lowest unoccupied molecular orbital (LUMO) -with a predominant contribution of the acceptor ligand- (LL'CT= ligand-to ligand CT with the coordinated metal acting as a $\pi$-bridge for the electron transfer). This CT process produces a decrease of the dipole moment from the ground to the excited state and accordingly negative solvatochromism and first hyperpolarizability $(\beta)$ is observed. 
S4. DATA FROM TD-DFT CALCULATIONS

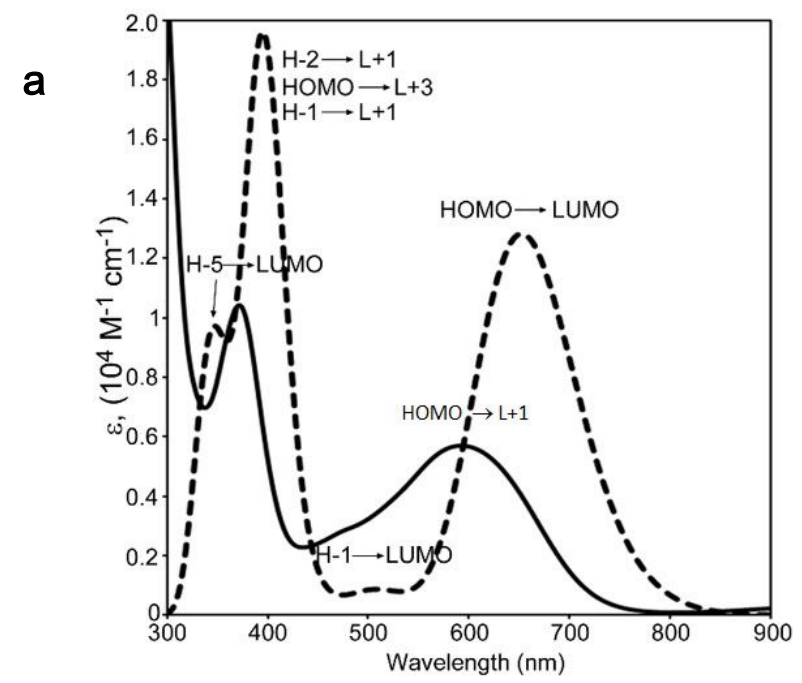

b
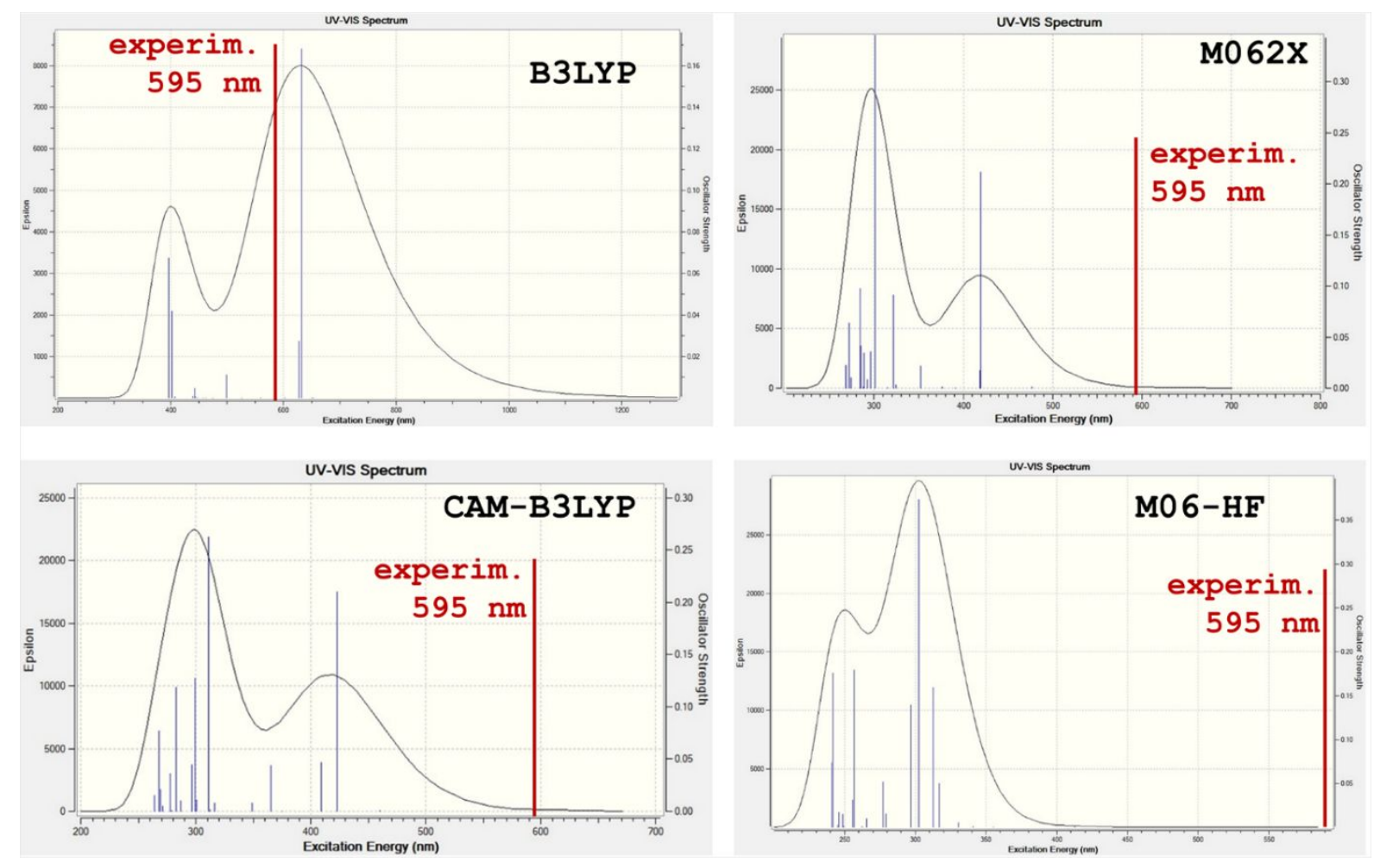

Figure S2: a, Experimental (solid line) and calculated (dashed line) absorption spectra for (1) in acetonitrile solution. Refer to Table S2 and Figure S3 for DFT Calculated MOs involved in the absorption transitions. b, Comparison between the absorption spectra 
computed using different density functionals (B3LYP, CAM-B3LYP, M06-2X, M06$\mathrm{HF})^{4,5}$ together with the experimental absorption (red line). See Figure $S 4 b$ for the comparison between the B3LYP, CAM-B3LYP, M06-2X, and M06-HF GS optimized geometry

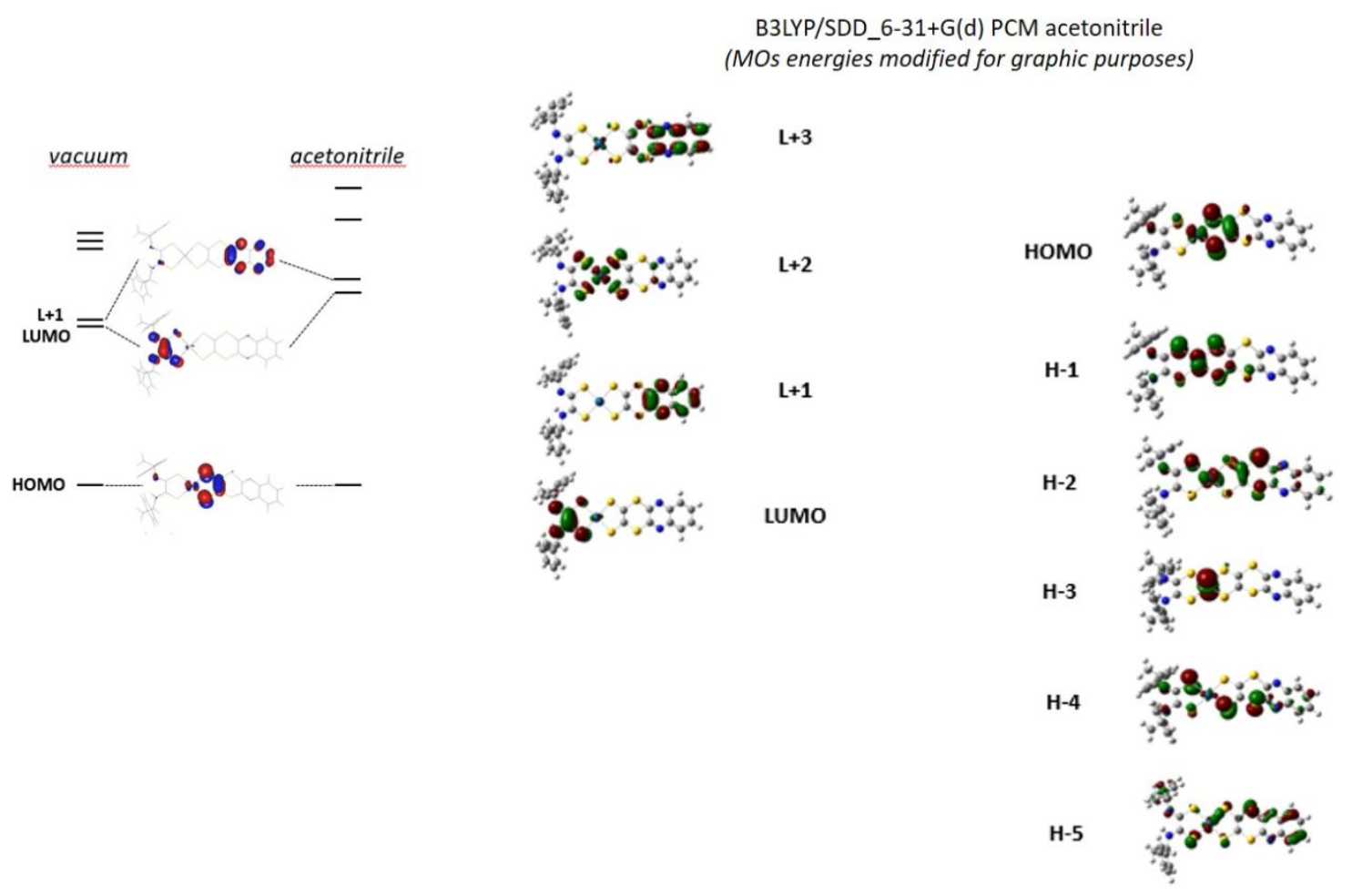

Figure S3: MOs of (1) from DFT methods. Left, comparison between the MOs energy levels from calculations performed in vacuum and in presence of acetonitrile as solvent (PCM method). Right, shape of the frontier MOs. 
Table S2: TD-DFT calculated energies and compositions of the lowest lying singlet electronic transitions of [Pt(MBAdto)(Quinoxdt)] (1) in the solution phase, acetonitrile, (B3LYP/6-31+G(d)-SDD). The principal singlet transition responsible for the first electronic absorption band is shown in bold, while the ones mainly responsible of the antiKasha emission are underlined.

\begin{tabular}{|l|l|l|l|l|}
\hline State & Composition $^{a}$ & $\Delta \mathrm{E}(\mathrm{eV} / \mathrm{nm}$ & $f^{c}$ & Character \\
\hline 1 & $\mathrm{HOMO} \rightarrow \mathrm{LUMO}, 97 \%$ & $1.90 / 653$ & 0.15 & Quinox/Pt $\rightarrow$ MBA \\
\hline$\underline{2}$ & $\underline{\mathrm{HOMO} \rightarrow \mathrm{L}+1,98 \%}$ & $\underline{1.96 / 632}$ & $\underline{0.0082}$ & $\underline{\text { Quinox/Pt } \rightarrow \text { Quinox }}$ \\
\hline 4 & $\underline{\mathrm{H}-1 \rightarrow \mathrm{LUMO}, 97 \%}$ & $\underline{2.44 / 508}$ & $\underline{0.011}$ & $\underline{\text { Quinox/Pt/MBA } \rightarrow \mathrm{MBA}}$ \\
\hline 5 & $\mathrm{H}-1 \rightarrow \mathrm{L}+2,77 \%$ & $2.78 / 446$ & 0.0001 & \\
\hline 6 & $\mathrm{H}-3 \rightarrow \mathrm{LUMO}, 93 \%$ & $2.81 / 441$ & 0.0006 & \\
\hline 7 & $\mathrm{HOMO} \rightarrow \mathrm{L}+3,64 \%$ & $3.03 / 409$ & 0.0021 & \\
\hline 8 & $\mathrm{H}-3 \rightarrow \mathrm{L}+2,28 \%$ & $2.80 / 444$ & 0.0050 & \\
\hline 9 & $\begin{array}{l}\mathrm{H}-3 \rightarrow \mathrm{L}+2,52 \% \\
\mathrm{H}-2 \rightarrow \mathrm{LUMO}, 22 \%\end{array}$ & $3.09 / 402$ & 0.029 & Pt $\rightarrow$ PtS 4 \\
\hline
\end{tabular}


a Compositions of electronic transitions are expressed in terms of contributing excitations between ground-state Kohn-Sham molecular orbitals.

${ }^{b}$ Transition energy from the ground state in $\mathrm{eV}$.

a
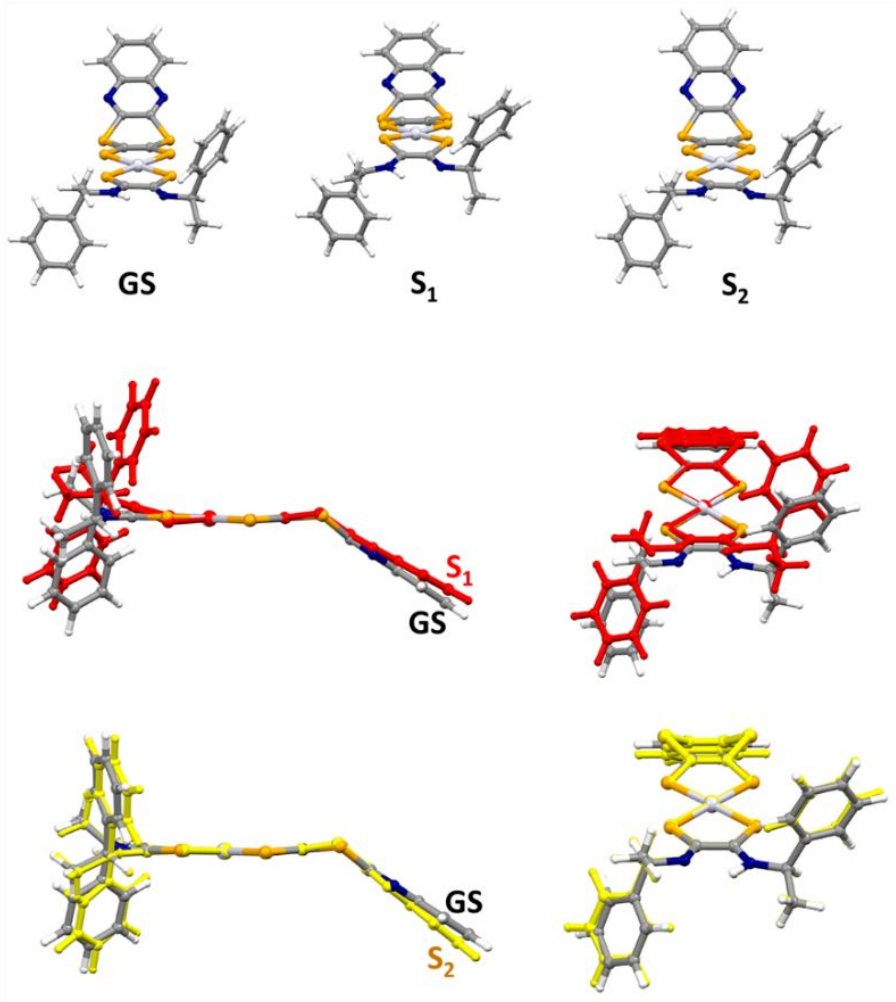
b
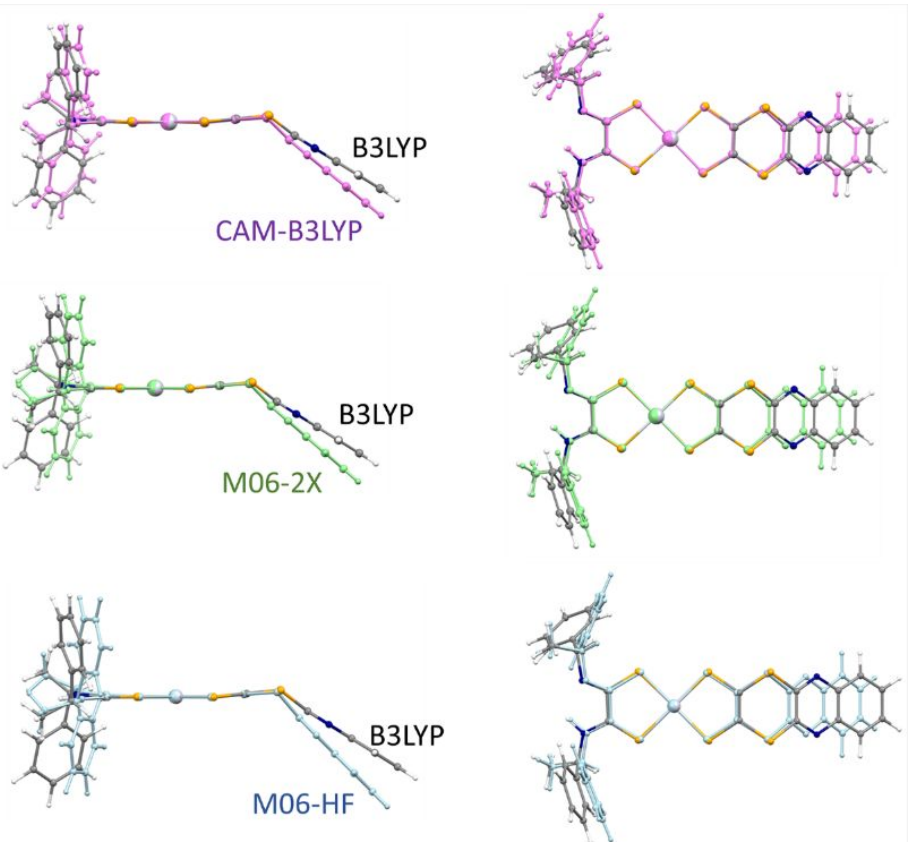

Figure S4: a, Optimized molecular geometries of the $G S$, and of the $S_{1}$ and $S_{2}$ states (B3LYP/6-31+G(d)-SDD). At the bottom, superposition of the optimized geometries of the GS (conventional atom color), with the singlet excited state (red), and of the second singlet excited state (yellow). b, Comparison between the B3LYP, CAMB3LYP, M06-2X, and M06-HF GS optimized geometry. 
Table S3: Calculated emission energies $(\Delta \mathrm{E})$ from excited states $S_{1}$ and $S_{2}$ for different optimized geometry. For sake of comparison, the corresponding data from Table S2 are shown in the last two rows (GS geometry).

\begin{tabular}{|c|c|c|c|c|}
\hline $\begin{array}{l}\text { Electronic state for } \\
\text { geometry optimization }\end{array}$ & Transition & $\begin{array}{l}\text { Main } \\
\text { Composition }\end{array}$ & $\begin{array}{l}\text { Transition } \\
\text { frequency } \\
(\Delta E)\end{array}$ & $\begin{array}{l}\text { Oscillator } \\
\text { strength }(\check{)}\end{array}$ \\
\hline$S_{1}$ & $\mathrm{~S}_{1} \rightarrow \mathrm{GS}$ & LUMO $\rightarrow$ HOMO & $\begin{array}{l}1.42 \mathrm{eV} \\
(872 \mathrm{~nm})\end{array}$ & 0.12 \\
\hline$S_{2}$ & $S_{1} \rightarrow G S$ & $\angle U M O \rightarrow H O M O$ & $\begin{array}{l}1.34 \mathrm{eV} \\
927 \mathrm{~nm}\end{array}$ & 0.0067 \\
\hline$S_{2}$ & $S_{2} \rightarrow G S$ & $\begin{array}{l}\angle U M O+1 \rightarrow \\
\text { HOMO }\end{array}$ & $\begin{array}{l}1.84 \mathrm{eV} \\
673 \mathrm{~nm}\end{array}$ & 0.17 \\
\hline$G S^{*}$ & $S_{1} \rightarrow G S^{*}$ & $\angle U M O \rightarrow H O M O$ & $\begin{array}{l}1.90 \mathrm{eV} \\
(653 \mathrm{~nm})\end{array}$ & 0.17 \\
\hline$G S^{*}$ & $S_{2} \rightarrow G S$ & $\begin{array}{l}\angle U M O+1 \rightarrow \\
\text { HOMO }\end{array}$ & $\begin{array}{l}1.96 \mathrm{eV} \\
632 \mathrm{~nm}\end{array}$ & 0.0082 \\
\hline
\end{tabular}

${ }^{*}$ From Table S2. 


\section{S5. DATA FROM TRANSIENT ABSORPTION EXPERIMENTS AT 615 NM}


Figure S5: a, 2D wavelength-time delay plot of TA experiment in acetonitrile upon

$615 \mathrm{~nm}$ excitation. The color code amplitude corresponds to differential absorbance (see legend). b, Same graph as Figure 2a, replotted for convenience of reader: representative selection of TA spectra at different time delays extracted from 2D TA time-wavelength experiment in panel a. c, Kinetics at selected probe wavelengths from data in panel a. 

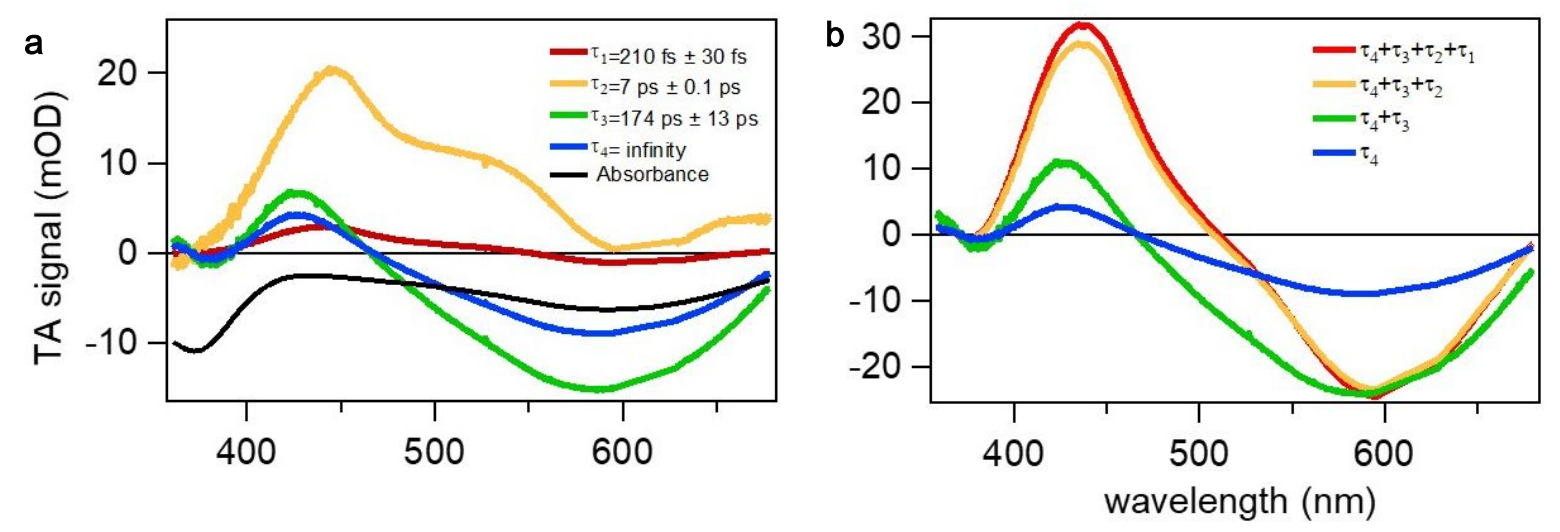

Figure S6: a, same graph as figure $2 b$, replotted for convenience of reader: DAS obtained by SVD analysis of TA experiment upon $615 \mathrm{~nm}$ excitation; each curve is labeled with the respective time constant. b, Sums of the DASs from panel A (or Figure $2 b)$ and representing the relaxation dynamics contribution in the overall signal of the subsequent lifetimes. Each curve is labelled with the respective sum.

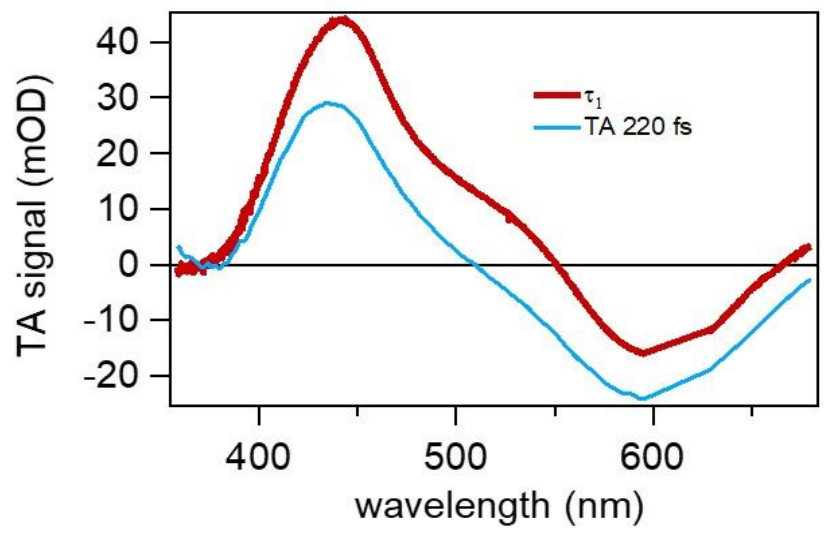


Figure S7: comparison of the first TA spectra at 220 fs with DAS $\mathrm{T}_{1}$, showing the same signal shape.

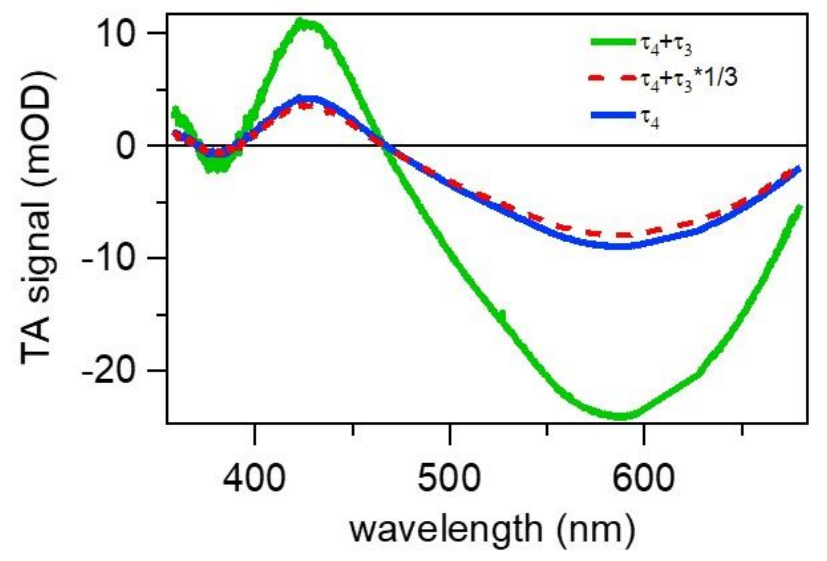

Figure S8: DAS $\mathrm{T}_{4}+\mathrm{T}_{3}$ and DAS $\mathrm{T}_{4}$ showing long timescale dynamics, confronted with DAS $T_{4}+T_{3}$ multiplied for the factor $1 / 3$, showing that the overall signal in $174 \mathrm{ps}$ is reduced of $2 / 3$.

Figure S5a shows a wavelength-time 2D plot of (1) in acetonitrile at subsequent pumpprobe TA time delays upon excitation of the lowest optical transition at $615 \mathrm{~nm}$. Figures S5b and c report a selection of TA spectra to better visualize the spectral evolution and kinetic traces to observe the dynamics, respectively.

Strong excited-state absorption (ESA) develops within the instrumental time resolution, characterized by a broad band around $435 \mathrm{~nm}$. The negative band centered at $600 \mathrm{~nm}$ closely matches the inverted steady-state absorption spectrum and it is therefore 
attributed to the ground-state bleaching (GSB). The bleaching at $380 \mathrm{~nm}$ is also attributed to GSB, though the signal is here dominated by the large ESA peaking at $435 \mathrm{~nm}$. In the first few hundreds of femtoseconds the overall signal has a first decay, afterwards most of the ESA decays in less than 20 ps, leaving a long-lasting component. Noteworthy is its spectrum that is slightly different from the initial one, in particular in the range 500 to 550 $\mathrm{nm}$ where an ESA signal disappears. Kinetic traces in Figure S5c due to positive and negative signals differ during the first 20 ps but follow the same temporal evolution at later times. By means of SVD data analysis, TA were spectrally decomposed into four decay associated spectra (DAS), shown in Figure S6a, each decaying with its own lifetime: 210 fs, $7 \mathrm{ps}, 174 \mathrm{ps}$ and $\infty$ (Table 1 of the main text).

The 210 fs DAS shows the same shape as the first TA spectra (Figure S7) and describes a very small signal decay (compare the red and orange curves in Figure S6A and B), revealing a small depopulation. The timescale is typical of fast internal vibrational energy redistribution and cooling, thus speaking for the presence of thermally activated non-radiative channel affecting the population in the sub-ps timescale. A time constant of $210 \mathrm{fs}$ is also characteristic of solvent response, which could modulate the electron density and thus the oscillator strength of the ESA band at $435 \mathrm{~nm}$ but not the GSB signal, in disagreement with the negative values of the $210 \mathrm{fs}$ DAS around $600 \mathrm{~nm}$ in Figure S6a. Since contributions due to stimulated emission can be ruled out because we excite at 615 $\mathrm{nm}$, we can conclude that the presence of thermally activated depopulation channels is the most reasonable rationale. 
The 7 ps DAS describes most of the decay of the TA signal of the ESA at $435 \mathrm{~nm}$ and a ground state recovery, as revealed by the negative band around $600 \mathrm{~nm}$ superimposed to a positive ESA signal. This agrees with a population decay of the $S_{1}$ state to the ground state. Since the overall signal is still always positive, there is an ESA signal overcoming the negative one of the GSB and responsible for the bands at 530 and at $680 \mathrm{~nm}$ (Figure S5B and Figure S6A). The lack of any spectral signature of these ESA bands in the longlived spectra reveals that this decay is accompanied by a change in the electronic state. Since the sample has a low emission $\Phi$, but we found that the excited state lifetime is on the ns timescale or longer, we can argue that the long-lived state is actually a triplet state. Accordingly, this component describes a signature of ISC from the first excited singlet to low-lying triplet states accompanied by non-radiative relaxation toward the GS. This assignment is corroborated by a recent ultrafast study on a closely related class of $d^{8}-$ metal D-M-A heteroleptic-dithiolene complexes, which reported an $\mathrm{S}_{1} \rightarrow \mathrm{T}_{1}$ ISC process with the same rate. ${ }^{6}$ Vibrational relaxation (VR) from hot $T_{1}$ state $\left({ }^{\#} T_{1}\right)$ towards the relaxed $\mathrm{T}_{1}$ state, due to internal vibrational relaxation and energy transfer to the solvent (cooling), should be observed but VR times are typically in the ps time scale or shorter and we cannot separate them from the pure ISC dynamics.

The third DAS component, with 174 ps lifetime, describes a decay of the overall signal by a factor $2 / 3$ (Figure S8) without any change in the spectral pattern. Both the 100 s of ps time scale and the spectral evolution is typical of rotational diffusion. ${ }^{6,7}$ Accordingly, we can safely assign this DAS to such a process. The last DAS corresponds to the 
differential absorption spectrum of a fully equilibrated $T_{1}$ state. The left side of Figure 3 shows the photocycle upon GS $\rightarrow S_{1}$ excitation (see also Figure $S 11 a$ ). 
S6. DATA FROM TRANSIENT ABSORPTION EXPERIMENTS AT 510 NM
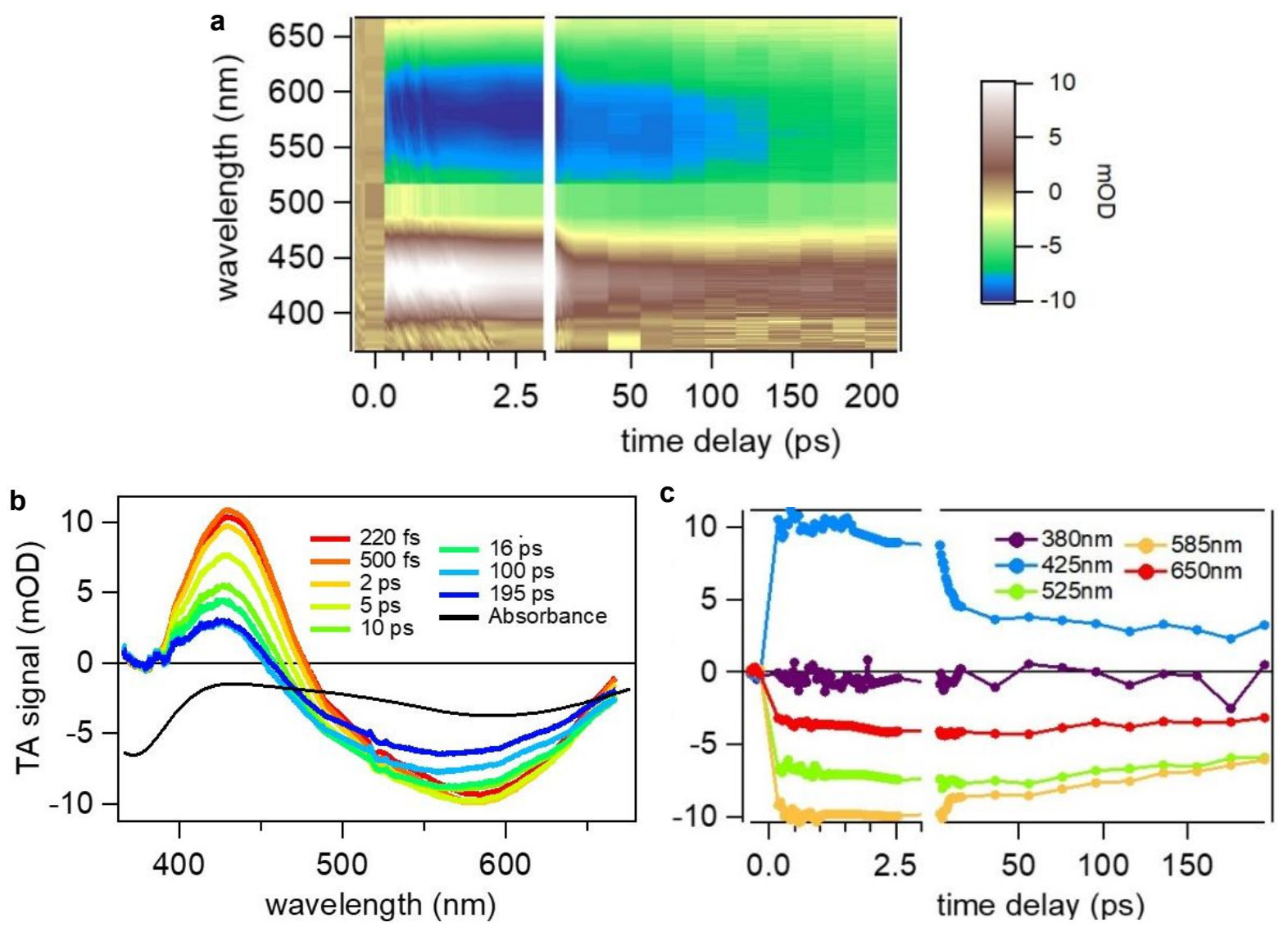

Figure S9. a, 2D wavelength-time delay plot of TA experiment in acetonitrile upon 510 $\mathrm{nm}$ excitation. The color code amplitude corresponds to differential absorbance (see legend). b, Same graph as Figure 2c, replotted for convenience of reader: representative selection of TA spectra at different time delays extracted from 2D TA time-wavelength experiment in panel a. c, Kinetics at selected probe wavelengths from data in panel a. 

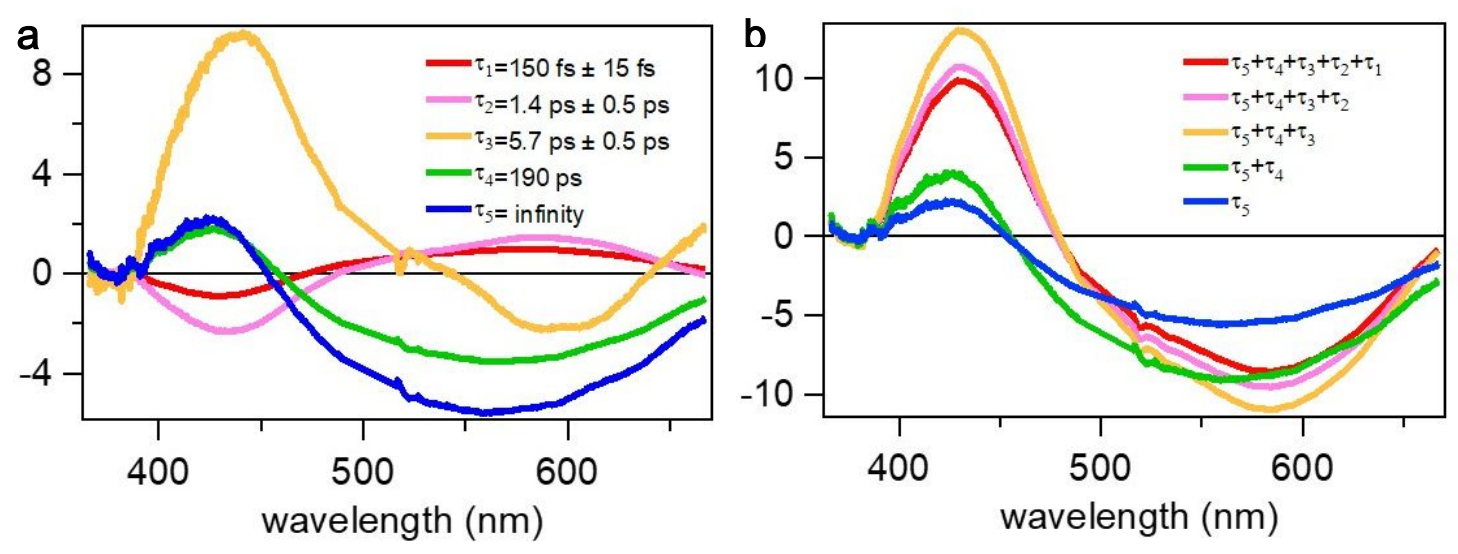

Figure S10: a, same graph as figure $2 \mathrm{~d}$, replotted for convenience of reader: DAS obtained by SVD analysis of TA experiment upon $510 \mathrm{~nm}$ excitation; each curve is labeled with the respective time constant. b, Sums of the DASs from panel a (or Figure 2d) and representing the relaxation dynamics contribution in the overall signal of the subsequent lifetimes. Each curve is labeled with the respective sum. 
a

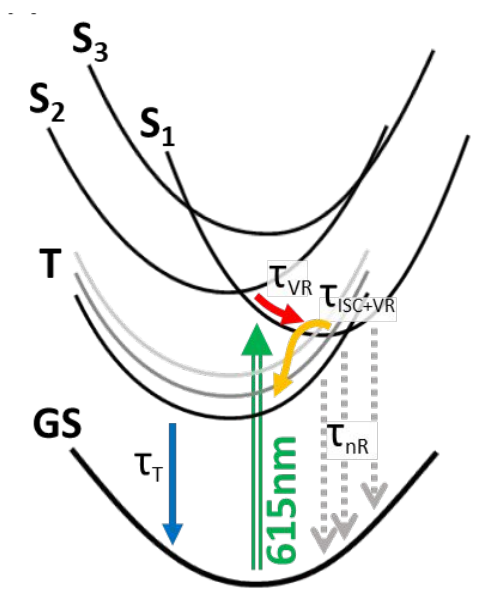

b

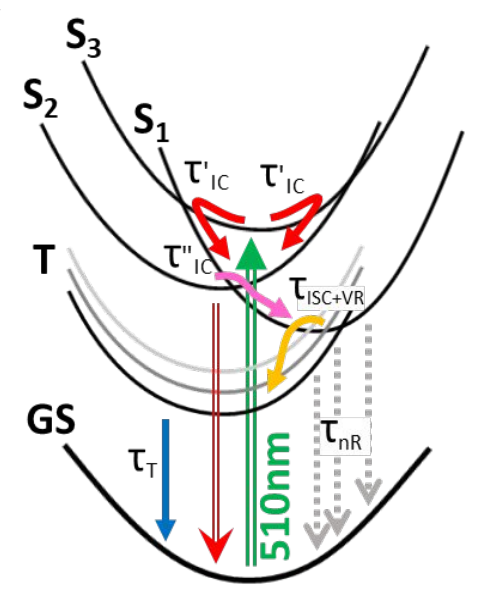

Figure S11: a, Photocycle upon $615 \mathrm{~nm}$ excitation into the lowest $\mathrm{S}_{1}$ excited state. $\mathbf{b}$, Photocycle upon $510 \mathrm{~nm}$ excitation into higher excited states $\left(S_{2}\right.$ and $\left.S_{3}\right)$. Arrows representing individual relaxation steps are color-coded according to the respective DASs in the left panel of Figure 5 and right panel of Figure 6 . See Table 1 in the manuscript for a summary of the time constant values. Notation for time constant labels: IC, internal conversion; VR, vibrational relaxation; ISC, intersystem crossing; T, triplet state. Electronic states nomenclature: $\mathbf{G S}$, ground state; $\mathbf{S}_{n}, \mathrm{n}^{\text {th }}$ singlet excited state; $\mathbf{T}$, low-lying triplet states. 
S8. COMPARISON BETWEEN TA AT $615 \mathrm{~nm}$ AND $510 \mathrm{~nm}$

To estimate the pure $S_{1}$ and $T_{1}$ spectra, without any contamination from the spectral changes due to the relaxation processes, we consider the sum of all the DAS components after the last relevant relaxation process, namely the sum of the last three DAS components for pure $S_{1}$ spectra (i.e. after the IC processes) and the last one for pure $T$ spectra (after ISC and rotational diffusion) (Figure 4). Spectra are rather close definitively supporting the proposed assignment (Figure S10b). The only major difference is observed in the spectral region from 480 to $600 \mathrm{~nm}$, where upon $510 \mathrm{~nm}$ excitation an ESA signal is missing or strongly reduced with respect to $615 \mathrm{~nm}$ excitation, as highlighted by the differential spectra in Figure 4 .

The lack of this band at any time (Figure 4a), in any relaxation process (Figure S13) and in particular in the spectra at the longest times (Figure 4b) strongly points to a long-lived distortion dependent on the initial excited state. The origin of the distortion can be conformational or electronic (a different final electronic state or ionization state), however, since all the main spectral features are preserved, we are currently in favor of a conformational distortion rather than having an electronic origin. This is also supported by the comparison of the optimized geometries of $G S, S_{1}$ and $S_{2}$ states (Figure S3), which confirms that the molecule undergoes important and global conformational changes upon inter-state transitions and suggests that the molecule is not rigid at all and it is prone to 
exploring different geometries during the relaxation. Interestingly, the molecular geometry of $G S$ is very similar to that of $S_{2}$ but it is significantly different from that of $S_{1}$.

It is worth noticing that this discussion on the origin of the conformational flexibility is based on the comparison of the geometries of the singlet excited states only. However, the existence of different conformers, and therefore the occurrence of anti-Kasha conformational photo-isomerization, is strictly related to the topology of the triplet excited state potential energy surfaces. Accordingly, in analogy with the analysis of the singlet states, we performed calculations to identify the equilibrated geometries of the triplet states involved in the photocycle, shown in Figure S13. This allowed us to verify that also the triplet states experience extensive conformational changes and show the same degree of flexibility of the singlet states. 




Figure S12: Comparison between the sum of the last three DASs from Figure $2 b$ and Figure 2d. This is equivalent to compare the orange curves from Figure S6 and in Figure S10. For sake of comparison, data are normalized to the maximum. Solid and dashed lines refer to $615 \mathrm{~nm}$ and $510 \mathrm{~nm}$ excitation, respectively. To isolate the ESA contribution dependent on the excitation wavelength, the difference spectrum is also plotted (grey line). The differential signal shows at $615 \mathrm{~nm}$ and $510 \mathrm{~nm}$ a distortion due to the excitation pulses. 
a
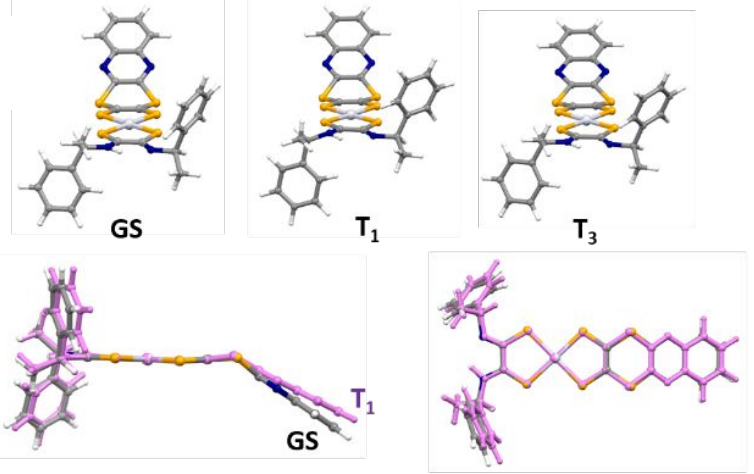

b
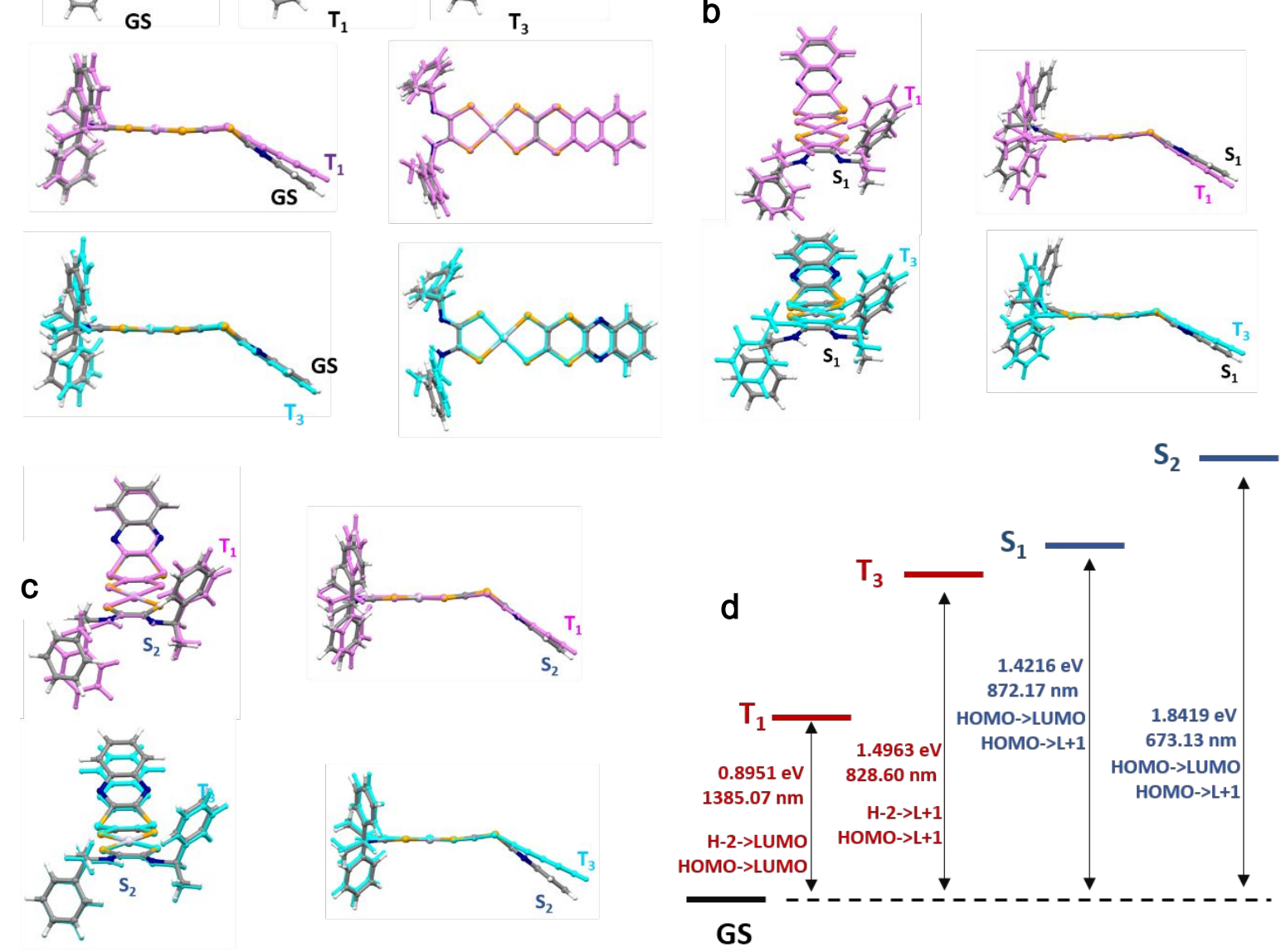

Figure S13: a, Comparison of the TD-DFT optimized molecular geometries between the ground state and the excited triplet states ( $T_{1}$ in pink and $T_{3}$ in blue). $b$, Comparison of the TD-DFT molecular geometries between the first excited singlet state and the excited triplet states. c, Comparison of the TD-DFT molecular geometries between the second excited singlet states and the excited triplet states. c, Diagram depicting equilibrium energies of the GS, of the triplet states $T_{1}$ and $T_{3}$, and of the singlet states $\mathrm{S}_{1}$ and $\mathrm{S}_{2}$. The excited state geometries were optimized using the TD-DFT method 
using the Tamm-Dancoff approximation. The vertical lines indicate the energy difference between the energy of the equilibrium excited geometries and the energy of the non-equilibrium geometry of the gound state (dashed line). MOs involved in the transitions are indicated. 
S9. COMPARISON WITH QUINOXDT CONTAINING MOLECULES
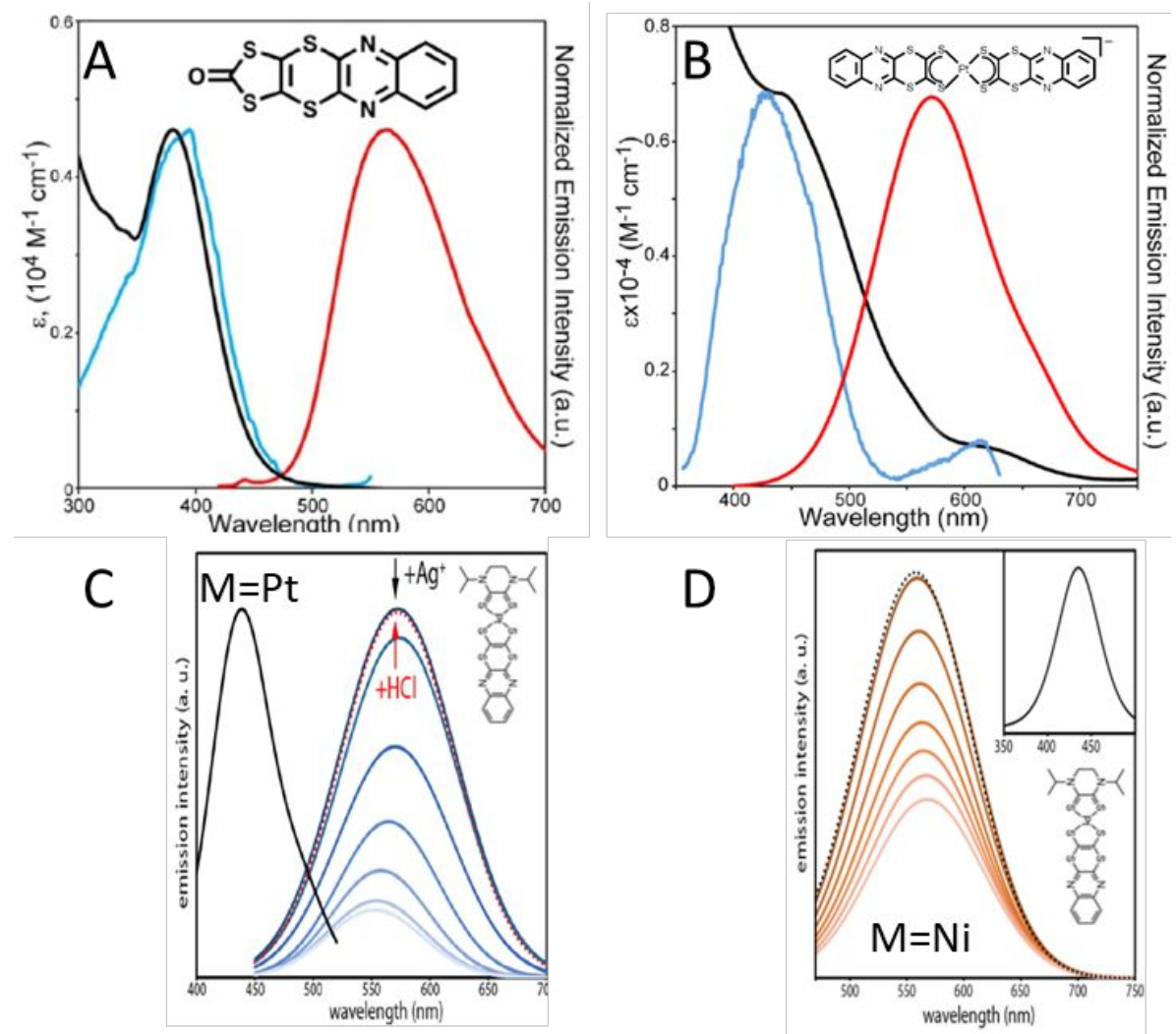

Figure S14: Comparison of anti-Kasha emission from Quinoxdt-bearing molecules including homo- and heterolepic dithiolene metal complexes with a ligand. The respective molecular structure are shown in each graph. a, b, Adapted from Ref. ${ }^{8}$ with the permission from the Royal Society of Chemistry. c, d, Adapted from Ref. ${ }^{9}$ with the permission from Wiley. 




Figure S15: Comparison of the anti-Kasha activity (PL and PLE spectra, solid and dashed line, respectively) of the heteroleptic Pt-Quinoxdt complex (1) herein investigated and another heteroleptic Pt-Quinoxdt complex from Ref. ${ }^{9}$ (labelled (2) and shown in Figure S14C) representative of the anti-Kasha behavior of a Pt-Quinoxdt complex bearing as acceptor ligand a neutral closed cyclic dithioxamide (red and blue lines, respectively). Other examples of Quinoxdt-bearing molecules are shown in Figure S14.

TD-DFT calculations (Table S3 and relative discussion) show that predictable emissions from the second excited state to the non-equilibrium $G S$ are centered at $673 \mathrm{~nm}$ with $f=0.17(\mathrm{LUMO}+1 \rightarrow \mathrm{HOMO})$ and $927 \mathrm{~nm} \mathrm{f}=0.0067$ (LUMO $\rightarrow \mathrm{HOMO}$ ), in satisfactory agreement with observed anti-Kasha emission at $720 \mathrm{~nm}$ and explaining the lack of any 
observed emission from $S_{1}$. Notably the anti-Kasha emission is rather red-shifted with respect to the emission of the sole Quinoxdt ligand and of other emissive Quinoxdt complexes, which show a recurring emission at 560-570nm (Figure S15). The comparison of the optimized structures of GS and the first two excited states (Figure S3) reveals major conformational changes involving the MBAdto counter-ligand, which are not expected in the other complexes. Indeed, due to its open structure, MBAdto is much more flexible than the counter-ligands of the other complexes, which are rigid cyclic groups (Figure S14). This higher flexibility on the one hand allows a deeper energy relaxation and therefore a greater Stokes shift; on the other hand, it permits the exploration of many more different conformational configurations, leading to long-lived conformers when relaxing from $S_{2}$ or $S_{3}$. 


\section{S10. COMPARISON OF THE $S_{2} \rightarrow S_{1}$ INTERNAL COVERSION AND $S_{2} \rightarrow G S$ LIFETIME WITH LITERATURE}

The values of $1.4 \mathrm{ps}$ for the $S_{2} \rightarrow S_{1}$ IC process and $22 \mathrm{~ns}$ for the $S_{2} \rightarrow G S$ radiative

lifetime should be compared with the 14 ps and 224 ns previously reported, respectively. ${ }^{2}$

This mismatch can be explained with the values of oscillator strength reported in Table

S2 and Table S3. Indeed, the oscillator strength for the $S_{2} \rightarrow G S$ emission is rather low

in the equilibrium configuration of GS, whereas it increases by one order of magnitude

upon relaxation toward the $S_{2}$-equilibrated configuration.

The value of $224 \mathrm{~ns}$ for the $S_{2} \rightarrow \mathrm{GS} \tau_{\text {rad }}$ (and therefore the $14 \mathrm{ps}$ for the $S_{2} \rightarrow S_{1}$ IC

process in Ref. 2) was calculated using the Strickler-Berg equation with steady-state

extinction coefficient, namely of the GS configuration. If the value in in Table S3 would

have been used, the expected value of $11 \mathrm{~ns}$ for the radiative lifetime and $0.7 \mathrm{ps}$ for the

$S_{2} \rightarrow S_{1}$ IC process would have been found. Considering the uncertainty of calculations

and the possibility that the system could not be fully relaxed to the equilibrated $S_{2}$

configuration in $\approx 1 \mathrm{ps}$ this value is in excellent agreement with the experimental one of 
$1.4 \mathrm{ps}$ and the estimation of $22 \mathrm{~ns}$ for $\mathrm{T}_{\text {rad }}$ reported in the main text. Indeed, estimating from the PLE spectrum in Figure 1 a bandwidth width of $60-70 \mathrm{~nm}$ at $450 \mathrm{~nm}(\Delta \omega / \omega=0.16)$, a radiative lifetime of 22 ns would correspond to an absorption amplitude $\varepsilon_{\text {Max }} \approx 700$ $\mathrm{M}^{-1} \mathrm{~cm}^{-1}$. The substantial consistency of these two independent estimations of the absorption cross section responsible for the anti-Kasha emission validates $22 \mathrm{~ns}$ as a good value for $\mathrm{T}_{\text {rad. }}$ It also corroborates the assignment of the anti-Kasha behavior to a competition between a relatively slow IC and radiative de-excitation from a higher emissive excited state (Figure 3 of the main text and Figure S11b).

It is worth mentioning that computational studies addressed to elucidate the role of the structure in optical properties of D-Pt-A model systems have recently shown through newly developed transition dipole decomposition methods that emissive states of these systems, where frontier orbitals are localized mostly on D or A, can even be converted from bright to dark depending on molecular distortions, which affect the transition dipolar moment between ground and excited states. ${ }^{9}$ 


\section{REFERENCES}

(1) Würth, C.; Grabolle, M.; Pauli, J.; Spieles, M.; Resch-Genger, U. Relative and Absolute Determination of Fluorescence Quantum Yields of Transparent Samples. Nature Protocols 2013, 8 (8), 1535-1550. https://doi.org/10.1038/nprot.2013.087.

(2) Attar, S.; Espa, D.; Artizzu, F.; Pilia, L.; Serpe, A.; Pizzotti, M.; di Carlo, G.; Marchiò, L.; Deplano, P. Optically Multiresponsive Heteroleptic Platinum-Dithiolene Complex with Proton Switchable Properties. Inorganic Chemistry 2017, 56 (12), 6763-6767.

(3) Ruckebusch, C., Silwa, M., Pernot, P., de Juan, A., Tauler, R. Comprehensive Data Analysis of Femtosecond Transient Absorption Spectra: A Review. Journal of Photochemistry and Photobiology C: Photochemistry Reviews 2012, 13(13), 1-27. https://doi.org/10.1016/j.jphotochemrev.2011.10.002.

Yanai, T.; Tew, D. P.; Handy, N. C. A New Hybrid Exchange-Correlation Functional Using the Coulomb-Attenuating Method (CAM-B3LYP). Chemical Physics Letters 2004, $393 \quad$ (1-3), 51-57. https://doi.org/10.1016/j.cplett.2004.06.011.

(5) Zhao, Y.; Truhlar, D. G. The M06 Suite of Density Functionals for Main Group Thermochemistry, Thermochemical Kinetics, Noncovalent Interactions, Excited States, and Transition Elements: Two New Functionals and Systematic Testing of Four M06-Class Functionals and 12 Other Functionals. Theoretical Chemistry Accounts 2008, 120 (1-3), 215-241. https://doi.org/10.1007/s00214-007-0310-x.

(6) Frei, F.; Rondi, A.; Espa, D.; Mercuri, M. L.; Pilia, L.; Serpe, A.; Odeh, A.; van Mourik, F.; Chergui, M.; Feurer, T. et al. Ultrafast Electronic and Vibrational Relaxations in Mixed-Ligand Dithione-Dithiolato Ni, Pd, and Pt Complexes. Dalton transactions (Cambridge, England: 2003) 2014, 43 (47), 17666-17676. https://doi.org/10.1039/c4dt01955e. 
(7) Lakowicz, J. R. Principles of Fluorescence Spectrosocpy, Springer, 2006. https://doi.org/10.1007/BF02629943.

(8) Attar, S.; Espa, D.; Artizzu, F.; Mercuri, M. L.; Serpe, A.; Sessini, E.; Concas, G.; Congiu, F.; Marchiò, L.; Deplano, P. A Platinum-Dithiolene Monoanionic Salt Exhibiting Multiproperties, Including Room-Temperature Proton-Dependent Solution Luminescence. Inorganic Chemistry 2016, 55 (11), 5118-5126. https://doi.org/10.1021/acs.inorgchem.5b02491.

(9) Attar, S. S.; Artizzu, F.; Marchiò, L.; Espa, D.; Pilia, L.; Casula, M. F.; Serpe, A.; Pizzotti, M.; Orbelli-Biroli, A.; Deplano, P. Uncommon Optical Properties and SilverResponsive Turn-Off/On Luminescence in a PtIl Heteroleptic Dithiolene Complex. $\begin{array}{lllll}\text { Chemistry 2018, } & 24 & \text { (Chemistry } & \text { A } & \text { E), }\end{array}$ https://doi.org/10.1002/chem.201801697. 